\title{
Convergence of a Generalized Pulse-Spectrum Technique (GPST) for Inverse Problems of 1-D Diffusion Equations in Space-Time Domain
}

\author{
By X. Y. Liu and Y. M. Chen
}

\begin{abstract}
The problem of convergence of a special form of the generalized pulsespectrum technique (GPST) for solving inverse problems of one-dimensional diffusion equations in space-time domain is considered. Under the assumptions that a Tikhonov regularized solution exists and the derivative operator of the regularized forward problem at the regularized solution is invertible, the iterative solutions of this special GPST converge to the Tikhonov regularized solution in $C$ norm if the initial guess is close enough to the Tikhonov regularized solution and the rate of convergence is at least linear.
\end{abstract}

1. Introduction. The generalized pulse-spectrum technique (GPST) [1] is a versatile and efficient iterative numerical algorithm for solving inverse problems of a system of nonlinear partial differential equations. In general, inverse problems of partial differential equations can be formulated as ill-posed nonlinear operator equations. It is important to point out that the GPST is not a single narrowly defined iterative numerical algorithm, but a broad class of iterative numerical algorithms based on the concept that either the nonlinear operator equation is first linearized by any one of the Newton-like iteration methods and then each iterate is solved by using a stabilizing method to overcome the instability, e.g., the Tikhonov regularization method [14]. Alternatively, the stabilizing method can be first applied to the nonlinear operator and then the stabilized nonlinear problem is solved by using a Newton-like iteration. Hence different choices of various Newton-like iteration methods and stabilizing methods lead to different special forms of GPST. The choice of a specific Newton-like iteration method and stabilizing method and the question of whether to solve the inverse problem in the space-time domain or in the space-complex frequency domain depend mainly on the particular inverse problem under consideration. The efficiency of a GPST depends upon how efficiently one can treat every single step in the particular numerical algorithm.

It has been demonstrated that the GPST iterative numerical algorithm does give very good results in solving the inverse problems with time-dependent coefficients of one-, two- and three-dimensional linear evolution partial differential equations in the space-complex frequency domain, [2]-[7], [10], [14], and in the space-time domain, [13]. Similarly, the inverse problems with time-dependent coefficients of a one-dimensional linear diffusion equation can be solved by using the GPST with equal efficacy [11]. The convergence of a special form of GPST for solving inverse

Received April 24, 1985; revised February 13, 1987.

1980 Mathematics Subject Classification (1985 Revision). Primary 35R30, 35R25. 
problems of one-dimensional evolutional partial differential equations in the spacecomplex frequency domain has been proved under several assumptions in [15].

In Section 2 a special form of GPST for solving one-dimensional diffusion equations in the space-time domain is introduced. This is followed by a section on the mathematical properties of the nonlinear inverse operator $A$ from $C[0,1]$ to $C[0, T]$. Finally, in Section 4 the convergence proof and error estimates are given. They show that if the initial guess $k_{0}(x)$ is close enough to the regularized solution $k_{\alpha}(x)$, then under the assumptions that a Tikhonov regularized solution exists and the derivative operator of the regularized forward problem at the regularized solution is invertible, the iterative sequence $\left\{k_{n}(x)\right\}, n=0,1,2,3, \ldots$, of this special form of GPST converges to $k_{\alpha}(x)$ and $\left\|k_{\alpha}-k_{n}\right\|_{C}<$ Const $\cdot \varsigma^{n}, 0<\varsigma<1$. The proof of the invertibility of the derivative operator of the regularized forward problem at the regularized solution will be presented elsewhere. It is clear that the generalization of this proof to cases of the corresponding inverse problems of higher spatial dimensions is rather straightforward but very tedious.

2. Generalized Pulse-Spectrum Technique (GPST). Consider the initialboundary value problem of the one-dimensional linear diffusion equation

$$
\begin{array}{ll}
\partial(k(x) \partial u / \partial x) / \partial x-\partial u / \partial t=0, & (x, t) \in Q=\{x \in(0,1), t \in(0, T)\} \\
u(x, 0)=0, & 0 \leq x \leq 1, \\
\partial u(0, t) / \partial x=f(t), & 0 \leq t \leq T, \\
u(1, t)=0, & 0 \leq t \leq T,
\end{array}
$$

and the auxiliary condition,

$$
u(0, t)=g(t), \quad 0 \leq t \leq T,
$$

where $k(x) \in \Sigma=\left\{k(x) \mid k(x) \in C[0,1], k_{-}<k(x)<k_{+}\right.$on $\left.[0,1]\right\}$ with constants $k_{-}, k_{+}>0$ and $k_{-} \leq 1, f(t) \in H^{1}(0, T), f(0)=0$ and $g(t) \in C[0, T], g(0)=0$, a function obtained from measurements.

The inverse problem here is to determine $k(x)$ such that $u(x, t)$ satisfies $(2.1)$ and (2.2). Mathematically, let there exist a nonlinear operator $A_{1}$ mapping $k(x) \rightarrow$ $u(x, t)$ and a trace operator $A_{2}$ mapping $u(x, t) \rightarrow g(t)$ on the proper part of the boundary. Hence the inverse problem amounts to solving the nonlinear operator equation,

$$
A \cdot k(x) \equiv A_{2} \cdot A_{1} \cdot k(x)=g(t) .
$$

Mathematically, linearizing (2.3) by a Newton-like iteration method first, and then solving each iterate by the Tikhonov regularization method, is similar to first applying the Tikhonov regularization method to (2.3) and then solving its corresponding Euler equation by a Newton-like iteration method. In actual computation, the first approach is more straightforward, but for theoretical analysis the second approach seems to be more convenient.

Based upon the second approach, the Tikhonov regularization method for solving (2.3) is to minimize the functional

$$
J_{\alpha}(k) \equiv\|A \cdot k-g\|_{L^{2}(0, T)}^{2}+\alpha^{2}\langle B \cdot k, k\rangle_{L^{2}[0,1]},
$$

where $k(x) \in \Sigma, B$ is a selfadjoint strictly positive bounded linear operator from $C[0,1]$ into its dual $V_{0}$ (the set of all regular functions of bounded variation on 
$x \in[0,1]$ vanishing at $x=0)$, and $\alpha^{2}$ is the regularization parameter. The Euler equation of $(2.4)$ is

$$
\phi(k) \equiv A^{\prime *}(k) \cdot(A \cdot k-g)+\alpha^{2} B \cdot k=0,
$$

where $A^{\prime}(k)$ is the Fréchet derivative of $A$ at $k$ and $A^{\prime *}(k)$ is the adjoint of $A^{\prime}(k)$.

For the special form of GPST here, the following Newton-like iteration method,

$$
k_{n+1}=k_{n}-N_{n}^{-1}\left[A^{\prime *}\left(k_{n}\right) \cdot\left(A \cdot k_{n}-g\right)+\alpha^{2} B \cdot k_{n}\right], \quad n=0,1,2,3, \ldots,
$$

with $N_{n} \equiv A^{\prime *}\left(k_{n}\right) \cdot A^{\prime}\left(k_{n}\right)+A^{\prime \prime *}\left(k_{0}\right) \cdot\left(A \cdot k_{0}-g\right)+\alpha^{2} B$ is used to solve the Euler equation (2.5).

3. Mathematical Properties of the Operator A. First, a basic lemma containing inequalities for integrals of the solution and its derivatives of an initialboundary value problem of a parabolic equation is proved. Next, the existence of a unique continuous solution of (2.1) for any $k(x) \in \Sigma$ and $f(t) \in H^{1}(0, T), f(0)=0$, is also proved, i.e., the operator $A$ is well defined. Finally, the Fréchet derivatives of $A$ of arbitrary order are obtained as the solutions of various initial-boundary value problems of parabolic equations.

Consider the set of functions $\Sigma_{\infty}=\Sigma \cap C^{\infty}[0,1]$, which is a dense subset of $\Sigma$ in the $C[0,1]$-norm, and the set $C^{\infty *}[0, T]=\left\{f(t) \mid f(t) \in C^{\infty}[0, T], f(0)=f^{(l)}(0)=\right.$ $0, l=1,2, \ldots\}$, which is a dense subset of $H^{1 *}(0, T)=\left\{f(t) \mid f(t) \in H^{1}(0, T)\right.$, $f(0)=0\}$ in the $H^{1}(0, T)$-norm.

\section{LEMMA 1. Let}

(i) $a(x) \in \Sigma_{\infty}, a_{i}(x) \in C^{\infty}[0,1], i=1,2, \ldots, m$,

(ii) $b(t) \in C^{\infty *}[0, T]$, and

(iii) $\psi_{i}(x, t) \in C^{\infty}(\mathbf{Q}), \psi_{i}(x, 0)=0, \partial \psi_{i}(0, t) / \partial x=c_{i}(t) \in C^{\infty *}[0, T], i=$ $1, \ldots, m$.

Then there exists a unique $C^{\infty}(\mathbf{Q})$ solution $\theta(x, t)$ of the following initial-boundary value problem:

$$
\begin{gathered}
\partial \theta / \partial t-\partial(a(x) \partial \theta / \partial x) / \partial x=\partial\left(\sum_{i=1}^{m} a_{i}(x) \partial \psi_{i} / \partial x\right) / \partial x, \quad(x, t) \in Q \\
\theta(x, 0)=0, \quad 0 \leq x \leq 1 \\
\partial \theta(0, t) / \partial x=b(t), \quad 0 \leq t \leq T \\
\theta(1, t)=0, \quad 0 \leq t \leq T
\end{gathered}
$$

Moreover, $\theta(x, t)$ satisfies

$$
\|\partial \theta / \partial x\|_{2, Q_{\tau}} \leq M_{0}
$$

$$
|\partial \theta / \partial t|_{2, \tau} \text { and }\left\|\partial^{2} \theta / \partial t \partial x\right\|_{2, Q_{\tau}} \leq M_{1}
$$$$
|\partial \theta / \partial x|_{2, \tau} \quad \text { and } \quad\|\theta\|_{C(\mathbf{Q})} \leq\left(2 M_{0} M_{1}\right)^{1 / 2}
$$

for any $\tau \in[0, T]$, where

$$
\begin{gathered}
\left|a_{i}\right|_{0}=\operatorname{Max}_{x \in[0,1]}\left|a_{i}(x)\right| \\
|\theta|_{2, \tau}^{2}=\int_{0}^{1} \theta^{2}(x, \tau) d x, \quad\|\theta\|_{2, Q_{\tau}}^{2}=\int_{0}^{\tau} \int_{0}^{1} \theta^{2}(x, t) d x d t
\end{gathered}
$$


and

$$
M_{0}=k_{-}^{-1}\left\{k_{+}\|b\|_{L^{2}(0, T)}+\sum_{i=1}^{m}\left|a_{i}\right|_{0}\left(\left\|c_{i}\right\|_{L^{2}(0, T)}+\left\|\partial \psi_{i} / \partial x\right\|_{2, Q_{\tau}}\right)\right\}
$$

$$
M_{1}=k_{-}^{-1}\left\{k_{+}\left\|b^{\prime}\right\|_{L^{2}(0, T)}+\sum_{i=1}^{m}\left|a_{i}\right|_{0}\left(\left\|c_{i}^{\prime}\right\|_{L^{2}(0, T)}+\left\|\partial^{2} \psi_{i} / \partial t \partial x\right\|_{2, Q_{\tau}}\right)\right\} .
$$

Proof. The proof of the existence of a $C^{\infty}(\mathbf{Q})$ solution of (3.1) can be found in [8]. To prove (3.2), multiply the first equation of (3.1) by $\theta(x, t)$ and integrate by parts on $\mathbf{Q}_{\tau}=\mathbf{Q} \cap[0, \tau]$ for $\tau \in[0, T]$, to obtain

$$
\begin{aligned}
\frac{1}{2}|\theta|_{2, \tau}^{2}+ & \left\|a^{1 / 2} \partial \theta / \partial x\right\|_{2, Q_{\tau}}^{2} \\
= & -\int_{0}^{\tau}\left[a(0) b(t)+\sum_{i=1}^{m} a_{i}(0) c_{i}(t)\right] \theta(0, t) d t \\
& -\int_{0}^{\tau} \int_{0}^{1} \sum_{i=1}^{m} a_{i}(x) \partial \psi_{i} / \partial x \cdot \partial \theta / \partial x d x d t \\
= & \int_{0}^{\tau} \int_{0}^{1}\left[a(0) b(t)+\sum_{i=1}^{m} a_{i}(0) c_{i}(t)-\sum_{i=1}^{m} a_{i}(x) \partial \psi_{i} / \partial x\right] \partial \theta / \partial x d x d t \\
\leq & \frac{1}{2}\left\|a^{1 / 2} \partial \theta / \partial x\right\|_{2, Q_{\tau}}^{2} \\
& +\frac{1}{2} \int_{0}^{\tau} \int_{0}^{1} a^{-1}(x)\left[a(0) b(t)+\sum_{i=1}^{m} a_{i}(0) c_{i}(t)-\sum_{i=1}^{m} a_{i}(x) \partial \psi_{i} / \partial x\right]^{2} d x d t .
\end{aligned}
$$

Consequently,

$$
\begin{aligned}
|\theta|_{2, \tau}^{2} & +\left\|a^{1 / 2} \partial \theta / \partial x\right\|_{2, Q_{\tau}}^{2} \\
& \leq k_{-}^{-1}\left\{k_{+}\|b\|_{L^{2}(0, T)}+\sum_{i=1}^{m}\left|a_{i}\right|_{0}\left(\left\|c_{i}\right\|_{L^{2}(0, T)}+\left\|\partial \psi_{i} / \partial x\right\|_{2, Q_{\tau}}\right)\right\}^{2}
\end{aligned}
$$

Inequality (3.2) now follows.

Let $\sigma(x, t)=\partial \theta(x, t) / \partial t$. This satisfies the initial-boundary value problem,

$$
\begin{gathered}
\partial \sigma / \partial t-\partial(a(x) \partial \sigma / \partial x) / \partial x=\partial\left(\sum_{i=1}^{m} a_{i}(x) \partial^{2} \psi_{i} / \partial t \partial x\right) / \partial x, \quad(x, t) \in Q \\
\sigma(x, 0)=\partial\left(\begin{array}{cl}
\left.a(x) \partial \theta / \partial x+\sum_{i=1}^{m} a_{i}(x) \partial \psi_{i} / \partial x\right) /\left.\partial x\right|_{t=0}=0, & 0 \leq x \leq 1 \\
\partial \sigma(0, t) / \partial x=b^{\prime}(t), & 0 \leq t \leq T, \text { and } \\
\sigma(1, t)=0, & 0 \leq t \leq T .
\end{array}\right.
\end{gathered}
$$

Upon comparing (3.1) with (3.5) one concludes that $\sigma(x, t)$ satisfies the estimation

$$
\begin{aligned}
|\sigma|_{2, \tau}^{2} & +\left\|a^{1 / 2} \partial \sigma / \partial x\right\|_{2, Q_{\tau}}^{2} \\
& \leq k_{-}^{-1}\left\{k_{+}\left\|b^{\prime}\right\|_{L^{2}(0, T)}+\sum_{i=1}^{m}\left|a_{i}\right|_{0}\left(\left\|c^{\prime}\right\|_{L^{2}(0, T)}+\left\|\partial^{2} \psi_{i} / \partial t \partial x\right\|_{2, Q_{\tau}}\right)\right\}^{2} \\
& =k_{-} M_{1}^{2} .
\end{aligned}
$$

Hence (3.3) is true. Finally, multiplying the first equation of $(3.1)$ by $\partial \theta(x, t) / \partial t$, integrating by parts on $\mathbf{Q}_{\tau}$, and following a similar procedure, one obtains (3.4). 
LEMMA 2. For any $k(x) \in \Sigma_{\infty}$ and $f(t) \in C^{\infty *}[0, T]$ there exists a unique $C^{\infty}(\mathbf{Q})$ solution $u(x, t)$ of $(2.1)$ which satisfies

$$
\|\partial u / \partial x\|_{2, Q_{\tau}} \leq k_{+} k_{-}^{-1}\|f\|_{L^{2}(0, T)},
$$

$$
|\partial u / \partial t|_{2, \tau} \text { and }\left\|\partial^{2} u / \partial t \partial x\right\|_{2, Q_{\tau}} \leq k_{+} k_{-}^{-1}\left\|f^{\prime}\right\|_{L^{2}(0, T)}
$$

$$
|\partial u / \partial x|_{2, \tau} \text { and }\|u\|_{C(\mathbf{Q})} \leq 2^{1 / 2} k_{+} k_{-}^{-1}\|f\|_{H^{1}(0, T)}
$$

for any $\tau \in[0, T]$. Moreover, for any $k^{*}(x), k(x) \in \Sigma_{\infty}$ and $f^{*}(t), f(t) \in C^{\infty *}[0, T]$, denote by $u^{*}(x, t)$ and $u(x, t)$ the solutions of (2.1) corresponding to $\left\{k^{*}, f^{*}\right\}$ and $\{k, f\}$, respectively; then their difference $u^{*}(x, t)-u(x, t)$ satisfies

$$
\begin{gathered}
\left\|\partial\left(u^{*}-u\right) / \partial x\right\|_{2, Q_{\tau}} \leq \frac{k_{-}+k_{+}}{k_{-}^{2}}\left\|f^{*}\right\|_{L^{2}(0, T)}\left|k^{*}-k\right|_{0}+k_{+} k_{-}^{-1}\left\|f^{*}-f\right\|_{L^{2}(0, T)} \\
\left|\partial\left(u^{*}-u\right) / \partial t\right|_{2, \tau} \text { and }\left\|\partial^{2}\left(u^{*}-u\right) / \partial t \partial x\right\|_{2, Q_{\tau}} \\
\leq\left.\frac{k_{-}+k_{+}}{k_{-}^{2}}\left\|f^{*}\right\|\right|_{L^{2}(0, T)}\left|k^{*}-k\right|_{0}+k_{+} k_{-}^{-1}\left\|f^{*}-f^{\prime}\right\|_{L^{2}(0, T)} \\
\left|\partial\left(u^{*}-u\right) / \partial x\right|_{2, \tau} \text { and }\left\|u^{*}-u\right\|_{C(\mathbf{Q})} \\
\leq 2^{1 / 2}\left[\frac{k_{-}+k_{+}}{k_{-}^{2}}\left\|f^{*}\right\|_{H^{1}(0, T)}\left|k^{*}-k\right|_{0}+k_{+} k_{-}^{-1}\left\|f^{*}-f\right\|_{H^{1}(0, T)}\right]
\end{gathered}
$$

for any $\tau \in[0, T]$.

Proof. This lemma can be proved by using Lemma 1 for the direct problem (2.1) and the initial-boundary value problem satisfied by the difference $u^{*}(x, t)-$ $u(x, t)$.

LEMMA 3. For any $k(x) \in \Sigma_{\infty}, h_{i}(x) \in C^{\infty}[0,1], i=1,2,3, \ldots, f(t) \in$ $C^{\infty *}[0, T]$, and any positive integer $l$, there exists a unique $C^{\infty}(\mathbf{Q})$ solution $w_{1,2, \ldots, l}(x, t)$ of the initial-boundary value problem,

$$
\begin{aligned}
& \partial w_{1,2, \ldots, l} / \partial t-\partial\left(k(x) \partial w_{1,2, \ldots, l} / \partial x\right) / \partial x \\
& =\partial\left(\sum_{i=1}^{l} h_{i}(x) \partial w_{1,2, \ldots, \boldsymbol{z}_{, \ldots, l}} / \partial x\right) / \partial x, \quad(x, t) \in Q \\
& w_{1,2, \ldots, l}(x, 0)=0, \quad 0 \leq x \leq 1, \\
& \partial w_{1,2, \ldots, l}(0, t) / \partial x=0, \quad w_{1,2, \ldots, l}(1, t)=0, \quad 0 \leq t \leq T
\end{aligned}
$$

where $w_{1,2, \ldots, \dot{\boldsymbol{x}}, \ldots, l} \equiv w_{1,2, \ldots, i-1, i+1, \ldots, l}, w_{\boldsymbol{k}}(0, t)=u(0, t)$ and $\partial w_{\boldsymbol{k}}(0, t) / \partial x=f(t)$. $w_{1,2, \ldots, l}(x, t)$ satisfies

$$
\left\|\partial w_{1,2, \ldots, l} / \partial x\right\|_{2, Q_{\tau}} \leq\left.\left.\frac{l !\left(k_{-}+k_{+}\right)}{k_{-}^{l+1}}\|f\|_{L^{2}(0, T)}\left|h_{1}\right|_{0}\right|_{2}\right|_{0} \cdots\left|h_{l}\right|_{0}
$$

$$
\begin{aligned}
& \left|\partial w_{1,2, \ldots, l} / \partial t\right|_{2, \tau} \text { and }\left\|\partial^{2} w_{1,2, \ldots, l} / \partial t \partial x\right\|_{2, Q_{\tau}} \\
& \quad \leq \frac{l !\left(k_{-}+k_{+}\right)}{k_{-}^{l+1}}\left\|f^{\prime}\right\|_{L^{2}(0, T)}\left|h_{1}\right|_{0}\left|h_{2}\right|_{0} \cdots\left|h_{l}\right|_{0}
\end{aligned}
$$




$$
\begin{aligned}
& \left|\partial w_{1,2, \ldots, l} / \partial x\right|_{2, \tau} \text { and }\left\|w_{1,2, \ldots, l}\right\|_{C(\mathbf{Q})} \\
& \quad \leq \frac{2^{1 / 2} l !\left(k_{-}+k_{+}\right)}{k_{-}^{l+1}}\|f\|_{H^{1}(0, T)}\left|h_{1}\right|_{0}\left|h_{2}\right|_{0} \cdots\left|h_{l}\right|_{0}
\end{aligned}
$$

for any $\tau \in[0, T]$ and $l=1,2,3, \ldots$ Moreover, for any $k^{*}(x)$ and $k(x) \in \Sigma_{\infty}$, $h_{i}^{*}(x)$ and $h_{i}(x) \in C^{\infty}[0,1], i=1,2, \ldots, f^{*}(t)$ and $f(t) \in C^{\infty *}[0, T]$, and any positive integer $l$, denote by $w_{1,2, \ldots, l}^{*}(x, t)$ and $w_{1,2, \ldots, l}(x, t)$ the solutions of $(3.12)$ corresponding to $\left\{k^{*}, h_{1}^{*}, h_{2}^{*}, \ldots, h_{l}^{*}, f^{*}\right\}$ and $\left\{k, h_{1}, h_{2}, \ldots, h_{l}, f\right\}$, respectively; then their difference $w_{1,2, \ldots, l}^{*}(x, t)-w_{1,2, \ldots, l}(x, t)$ satisfies

$$
\begin{aligned}
\left\|\partial\left(w_{1, \ldots, l}^{*}-w_{1, \ldots, l}\right) / \partial x\right\|_{2, Q_{\tau}} & \\
\leq & \frac{(l+1) !\left(k_{-}+k_{+}\right)}{k_{-}^{l+2}} F_{0} H_{1} H_{2} \cdots H_{l} \\
& \cdot\left\{\left|k^{*}-k\right|_{0}+\frac{k_{-}}{l+1}\left(\sum_{i=1}^{l} \frac{\left|h_{i}^{*}-h_{i}\right|_{0}}{H_{i}}+\frac{\left\|f^{*}-f\right\|_{L^{2}(0, T)}}{F_{0}}\right)\right\}, \\
\left|\partial\left(w_{1, \ldots, l}^{*}-w_{1, \ldots, l}\right) / \partial t\right|_{2, \tau} \text { and }\left\|\partial^{2}\left(w_{1, \ldots, l}^{*}-w_{1, \ldots, l}\right) / \partial t \partial x\right\|_{2, Q_{\tau}} & (l+1) !\left(k_{-}+k_{+}\right) \\
\leq & \frac{k_{-}^{l+2}}{F_{1}} H_{1} H_{2} \cdots H_{l} \\
& \cdot\left\{\left|k^{*}-k\right|_{0}+\frac{k_{-}}{l+1}\left(\sum_{i=1}^{l} \frac{\left|h_{i}^{*}-h_{i}\right|_{0}}{H_{i}}+\frac{\left\|f^{*}-f^{\prime}\right\|_{L^{2}(0, T)}}{F_{1}}\right)\right\}, \\
\left|\partial\left(w_{1, \ldots, l}^{*}-w_{1, \ldots, l}\right) / \partial x\right|_{2, \tau} \text { and }\left\|w_{1, \ldots, l}^{*}-w_{1, \ldots, l}\right\|_{C(\mathbf{Q})} & \frac{2^{1 / 2}(l+1) !\left(k_{-}+k_{+}\right)}{k_{-}^{l+2}}\left(F_{0}+F_{1}\right) H_{1} \cdots H_{l} \\
\leq & \cdot\left\{\left|k^{*}-k\right|_{0}+\frac{k_{-}}{l+1}\left(\sum_{i=1}^{l} \frac{\left|h_{i}^{*}-h_{i}\right|_{0}}{H_{i}}+\frac{\left\|f^{*}-f\right\|_{H^{1}(0, T)}}{F_{0}+F_{1}}\right)\right\}
\end{aligned}
$$

for any $\tau \in[0, T]$ and $l=1,2,3, \ldots$ Here the positive constants $F_{0}, F_{1}, H_{1}, H_{2}, \ldots$ are chosen such that

$$
\begin{aligned}
& \operatorname{Max}\left\{\left\|f^{*}\right\|_{L^{2}(0, T)},\|f\|_{L^{2}(0, t)}\right\} \leq F_{0}, \\
& \operatorname{Max}\left\{\left\|f^{*}\right\|_{L^{2}(0, T)},\left\|f^{\prime}\right\|_{L^{2}(0, T)}\right\} \leq F_{1}, \\
& \operatorname{Max}\left\{\left|h_{i}^{*}\right|_{0},\left|h_{i}\right|_{0}\right\} \leq H_{i}, \quad i=1,2,3, \ldots
\end{aligned}
$$

Proof. Applying Lemma 1 and Lemma 2 to (3.12) and to the initial-boundary value problem for $w_{1,2, \ldots, l}^{*}(x, t)-w_{1,2, \ldots, l}(x, t)$, and using induction on $l$, the desired inequalities follow after some tedious manipulation.

Remark. For the special case of $h_{i}^{*}(x)=h_{i}(x), i=1,2,3, \ldots$, and $f^{*}(t)=f(t)$, Eqs. (3.16), (3.17) and (3.18) become, respectively,

$$
\begin{aligned}
& \left\|\partial\left(w_{1, \ldots, l}^{*}-w_{1, \ldots, l}\right) / \partial x\right\|_{2, Q_{\tau}} \\
& \quad \leq \frac{(l+1) !\left(k_{-}+k_{+}\right)}{k_{-}^{l+2}}\|f\|_{L^{2}(0, T)}\left|h_{1}\right|_{0} \cdots\left|h_{l}\right|_{0}\left|k^{*}-k\right|_{0}, \\
& \left|\partial\left(w_{1, \ldots, l}^{*}-w_{1, \ldots, l}\right) / \partial t\right|_{2, \tau} \text { and }\left\|\partial^{2}\left(w_{1, \ldots, l}^{*}-w_{1, \ldots, l}\right) / \partial t \partial x\right\|_{2, Q_{\tau}} \\
& \quad \leq \frac{(l+1) !\left(k_{-}+k_{+}\right)}{k_{-}^{l+2}}\left\|f^{\prime}\right\|_{L^{2}(0, T)}\left|h_{1}\right|_{0}\left|h_{2}\right|_{0} \cdots\left|h_{l}\right|_{0}\left|k^{*}-k\right|_{0},
\end{aligned}
$$




$$
\begin{aligned}
& \left|\partial\left(w_{1, \ldots, l}^{*}-w_{1, \ldots, l}\right) / \partial x\right|_{2, \tau} \text { and }\left\|w_{1, \ldots, l}^{*}-w_{1, \ldots, l}\right\|_{C(\mathbf{Q})} \\
& \quad \leq \frac{2^{1 / 2}(l+1) !\left(k_{-}+k_{+}\right)}{k_{-}^{l+2}}\|f\|_{H^{1}(0, T)}\left|h_{1}\right|_{0}\left|h_{2}\right|_{0} \cdots\left|h_{l}\right|_{0}\left|k^{*}-k\right|_{0} .
\end{aligned}
$$

LEMMA 4. For any $k^{*}(x), k(x) \in \Sigma_{\infty}, h_{i}(x) \in C^{\infty}[0,1], i=1,2, \ldots$, and $f(t) \in C^{\infty *}[0, T]$, the differences

$$
\begin{aligned}
& p_{1}(x, t)=u^{*}(x, t)-u(x, t)-w_{1}(x, t) \quad \text { and } \\
& p_{1,2, \ldots, l}(x, t)=w_{1, \ldots, l-1}^{*}(x, t)-w_{1, \ldots, l-1}(x, t)-w_{1, \ldots, l}(x, t) \\
& l=2,3, \ldots,
\end{aligned}
$$

satisfy

$$
\left\|p_{1}\right\|_{C(\mathbf{Q})} \leq \frac{2^{1 / 2}\left(k_{-}+k_{+}\right)}{k_{-}^{3}}\|f\|_{H^{1}(0, T)}\left|k^{*}-k\right|_{0}^{2}
$$

and

$$
\begin{array}{r}
\left\|p_{1, \ldots, l}\right\|_{C(\mathbf{Q})} \leq \frac{2^{1 / 2}(l+1) !\left(k_{-}+k_{+}\right)}{k_{-}^{l+2}}\|f\|_{H^{1}(0, T)}\left|h_{1}\right|_{0} \cdots\left|h_{l-1}\right|_{0}\left|k^{*}-k\right|_{0}^{2} \\
l=2,3,4, \ldots
\end{array}
$$

Here, $w_{1,2, \ldots, l-1}^{*}(x, t), w_{1,2, \ldots, l-1}(x, t)$ and $w_{1,2, \ldots, l}(x, t)$ are the solutions of $(3.12)$ corresponding to $\left\{k^{*}, h_{1}, \ldots, h_{l-1}, f\right\},\left\{k, h_{1}, \ldots, h_{l-1}, f\right\}$ and $\left\{k, h_{1}, \ldots, h_{l-1}, k^{*}-\right.$ $k, f\}$, respectively.

Proof. This lemma is a direct consequence of Lemma 1 and the remark after Lemma 3.

LEMMA 5. For all $k(x) \in \Sigma$ one has

$$
A \cdot k(x) \subset C[0, T] .
$$

Proof. For any fixed $k(x) \in \Sigma$ and $f(t) \in H^{1 *}(0, T)$ one can construct their approximate sequences $\left\{k_{n}(x)\right\} \subset \Sigma_{\infty}$ and $\left\{f_{n}(t)\right\} \subset C^{\infty *}[0, T]$ such that

$$
\left|k_{n}-k\right|_{0} \rightarrow 0, \quad\left\|f_{n}-f\right\|_{H^{1}(0, T)} \rightarrow 0 \quad \text { as } n \rightarrow \infty .
$$

Without loss of generality, let

$$
\left\|f_{n}\right\|_{L^{2}(0, T)} \leq\|f\|_{L^{2}(0, T)}+1 \equiv F_{0} \quad \text { and } \quad\left\|f_{n}^{\prime}\right\|_{L^{2}(0, T)} \leq\left\|f^{\prime}\right\|_{L^{2}(0, T)}+1 \equiv F_{1} .
$$

Consider the family of initial-boundary value problems

$$
\begin{aligned}
& \partial u_{n} / \partial t-\partial\left(k_{n}(x) \partial u_{n} / \partial x\right) / \partial x=0, \quad(x, t) \in Q \\
& u_{n}(x, 0)=0, \quad 0 \leq x \leq 1 \\
& \partial u_{n}(0, t) / \partial x=f_{n}(t), \quad 0 \leq t \leq T \\
& u_{n}(1, t)=0, \quad 0 \leq t \leq T
\end{aligned}
$$

Using the results of Lemma 1 for the $C^{\infty}(\mathbf{Q})$ solution of the above initial-boundary value problems and their differences $u_{n}(x, t)-u_{m}(x, t), m, n=1,2, \ldots$, one can 
derive the following estimates,

$$
\begin{gathered}
\left\|\partial u_{n} / \partial x\right\|_{2, Q_{\tau}} \leq k_{+} k_{-}^{-1}\left\|f_{n}\right\|_{L^{2}(0, T)} \leq k_{+} k_{-}^{-1} F_{0} \\
\left|\partial u_{n} / \partial t\right|_{2, \tau} \text { and }\left\|\partial^{2} u_{n} / \partial t \partial x\right\|_{2, Q_{\tau}} \leq k_{+} k_{-}^{-1}\left\|f_{n}^{\prime}\right\|_{L^{2}(0, T)} \leq k_{+} k_{-}^{-1} F_{1}, \\
\left|\partial u_{n} / \partial x\right|_{2, \tau} \text { and }\left\|u_{n}\right\|_{C(\mathbf{Q})} \leq 2^{1 / 2} k_{+} k_{-}^{-1}\left\|f_{n}\right\|_{H^{1}(0, T)} \leq 2^{1 / 2} k_{+} k_{-}^{-1}\left(F_{0}+F_{1}\right), \\
\left\|\partial\left(u_{n}-u_{m}\right) / \partial x\right\|_{2, Q_{\tau}} \leq\left(k_{-}+k_{+}\right) k_{-}^{-2} F_{0}\left|k_{n}-k_{m}\right|_{0}+k_{+} k_{-}^{-1}\left\|f_{n}-f_{m}\right\|_{L^{2}(0, T)}, \\
\left|\partial\left(u_{n}-u_{m}\right) / \partial t\right|_{2, \tau} \&\left\|\partial^{2}\left(u_{n}-u_{m}\right) / \partial t \partial x\right\|_{2, Q_{\tau}} \\
\leq \frac{k_{-}+k_{+}}{k_{-}^{2}} F_{1}\left|k_{n}-k_{m}\right|_{0}+\frac{k_{+}}{k_{-}}\left\|f_{n}^{\prime}-f_{m}^{\prime}\right\|_{L^{2}(0, T)}
\end{gathered}
$$

and

$$
\begin{aligned}
& \left|\partial\left(u_{n}-u_{m}\right) / \partial x\right|_{2, \tau} \&\left\|u_{n}-u_{m}\right\|_{C(\mathbf{Q})} \\
& \quad \leq 2^{1 / 2}\left\{\frac{k_{-}+k_{+}}{k_{-}^{2}}\left(F_{0}+F_{1}\right)\left|k_{n}-k_{m}\right|_{0}+\frac{k_{+}}{k_{-}}\left\|f_{n}-f_{m}\right\|_{H^{1}(0, T)}\right\} .
\end{aligned}
$$

From the above estimates it follows that there exists a function $u(x, t) \in C(\mathbf{Q})$ such that

$$
\begin{gathered}
u_{n}(x, t) \stackrel{C(\mathbf{Q})}{\longrightarrow} u(x, t), \\
\partial u_{n}(x, t) / \partial x \stackrel{L^{2}(0,1)}{\longrightarrow} \partial u(x, t) / \partial x, \quad \partial u_{n}(x, t) / \partial t \stackrel{L^{2}(0,1)}{\longrightarrow} \partial u(x, t) / \partial t
\end{gathered}
$$

for any $t \in|0, T|$, and

$$
\partial^{2} u_{n}(x, t) / \partial t \partial x \stackrel{L^{2}(\mathbf{Q})}{\longrightarrow} \partial^{2} u(x, t) / \partial t \partial x .
$$

Moreover, the norms of $u(x, t)$ and its derivatives also satisfy the above estimates.

Let $V=\left\{v(x, t) \mid v(x, t) \in C(\mathbf{Q}), \partial v / \partial x \in L^{2}(Q)\right.$, and $v(0, t)=0$ for $\left.t \in[0, T]\right\}$. It can be easily verified that $u_{n}(x, t)$ satisfies the integral identity

$$
\int_{0}^{\tau} \int_{0}^{1}\left\{\partial u_{n} / \partial t \cdot v+k_{n} \partial u_{n} / \partial x \cdot \partial v / \partial x\right\} d x d t+\int_{0}^{\tau} k_{n}(0) f_{n}(t) v(0, t) d t=0
$$

for any $v \in V$ and $\tau \in[0, T]$. Therefore, $u(x, t)$ satisfies the same integral identity with $n \rightarrow \infty$. This implies that $u(x, t)$ is a continuous solution of $(2.1)$ in the weak sense. From the uniqueness properties of weak solutions of linear parabolic equations, $u(x, t)$ is also a unique continuous solution of $(2.1)$. Thus, for any $k(x) \in$ $\Sigma$ and $f(t) \in H^{1 *}(0, T)$, one has $A_{1} \cdot k(x)=u(x, t) \in C(\mathbf{Q})$ and $A \cdot k(x)=u(0, t) \in$ $C[0, T]$.

Remark. It is obviously true that $u(0, t)=-\int_{0}^{1} \partial u(x, t) / \partial x d x$ for $t \in[0, T]$.

The following relation then exists between the operators $A_{1}$ and $A$ :

$$
A \cdot k(x)=-\int_{0}^{1} \partial\left(A_{1} \cdot k(x)\right) / \partial x d x \quad \text { for } t \in[0, T]
$$

LEMMA 6. The operator $A$ is Fréchet differentiable up to an arbitrary order and the lth-order Fréchet differential

$$
A^{(l)}(k) \cdot h_{1} h_{2} \cdots h_{l}=w_{1,2, \ldots, l}(0, t) \in C[0, T]
$$


for any $k \in \Sigma, h_{i} \in C[0,1], i=1,2, \ldots, l, l=1,2, \ldots$, and $f(t) \in H^{1 *}(0, T)$, where $w_{1,2, \ldots, l}(x, t)$ is the continuous solution of the initial-boundary value problem (3.12) corresponding to $\left\{k, h_{1}, h_{2}, \ldots, h_{l}, f\right\}$.

Proof. Let $k(x) \in \Sigma, h_{i}(x) \in C[0,1], i=1,2, \ldots$, and $f(t) \in H^{1 *}(0, T)$ be given. Let the approximate sequences $\left\{k_{n}(x)\right\} \subset \Sigma_{\infty},\left\{h_{i n}(x)\right\} \subset C^{\infty}[0,1], i=$ $1,2, \ldots$, and $\left\{f_{n}(t)\right\} \subset C^{\infty *}[0, T]$ satisfy the conditions in the proof of Lemma 5 : $\left|h_{\text {in }}-h_{i}\right|_{0} \rightarrow 0$ as $n \rightarrow \infty$ and $\left|h_{i n}\right|_{0} \leq\left|h_{i}\right|_{0}+1 \equiv H_{i}, i, n=1,2, \ldots$ Then one has the $C^{\infty}(\mathbf{Q})$ sequence solution $\left\{u_{n}(x, t)\right\}$ of $(2.1)$ and the $C^{\infty}(\mathbf{Q})$ sequence solutions $\left\{w_{1,2, \ldots, l, n}(x, t)\right\}$ of $(3.12), l=1,2,3, \ldots$ From Lemma 5 , the limit function $u(x, t)$ is the continuous solution of $(2.1)$, i.e., $A_{1} \cdot k(x)=u(x, t)$ for any $k(x) \in \Sigma$. Using the results of Lemma 2 for $\left\{w_{1,2, \ldots, l, n}(x, t)\right\}$, one obtains the following estimates:

$$
\begin{gathered}
\left\|\partial w_{1, \ldots, l, n} / \partial x\right\|_{2, Q_{\tau}} \leq \frac{l !\left(k_{-}+k_{+}\right)}{k_{-}^{l+1}} F_{0} H_{1} H_{2} \cdots H_{l} \\
\left|\partial w_{1, \ldots, l, n} / \partial t\right|_{2, \tau} \text { and }\left\|\partial^{2} w_{1, \ldots, l, n} / \partial t \partial x\right\|_{2, Q_{\tau}} \\
\leq \frac{l !\left(k_{-}+k_{+}\right)}{k_{-}^{l+1}} F_{1} H_{1} H_{2} \cdots H_{l} \\
\left|\partial w_{1, \ldots, l, n} / \partial x\right|_{2, \tau} \text { and }\left\|w_{1, \ldots, l, n}\right\|_{C(\mathbf{Q})} \\
\leq \frac{2^{1 / 2} l !\left(k_{-}+k_{+}\right)}{k_{-}^{l+1}}\left(F_{0}+F_{1}\right) H_{1} H_{2} \cdots H_{l} .
\end{gathered}
$$

Moreover,

$$
\begin{aligned}
&\left\|\partial\left(w_{1, \ldots, l, n}-w_{1,2, \ldots, l, m}\right) / \partial x\right\|_{2, Q_{\tau}} \\
& \leq \frac{(l+1) !\left(k_{-}+k_{+}\right)}{k_{-}^{l+2}} F_{0} H_{1} H_{2} \cdots H_{l} \\
& \cdot\left\{\left|k_{n}-k_{m}\right|_{0}+\frac{k_{-}}{l+1}\left(\sum_{i=1}^{l} \frac{\left|h_{i n}-h_{i m}\right|_{0}}{H_{i}}+\frac{\left\|f_{n}-f_{m}\right\|_{L^{2}(0, T)}}{F_{0}}\right)\right\} \\
&\left|\partial\left(w_{1, \ldots, l, n}-w_{1, \ldots, l, m}\right) / \partial t\right|_{2, \tau} \text { and }\left\|\partial^{2}\left(w_{1, \ldots, l, n}-w_{1, \ldots, l, m}\right) / \partial t \partial x\right\|_{2, Q_{\tau}} \\
& \leq \frac{(l+1) !\left(k_{-}+k_{+}\right)}{k_{-}^{l+2}} F_{1} H_{1} \cdots H_{l} \\
& \cdot\left\{\left|k_{n}-k_{m}\right|_{0}+\frac{k_{-}}{l+1}\left(\sum_{i=1}^{l} \frac{\left|h_{i n}-h_{i m}\right|_{0}}{H_{i}}+\frac{\left\|f_{n}^{\prime}-f_{m}^{\prime}\right\|_{L^{2}(0, T)}}{F_{1}}\right)\right\}
\end{aligned}
$$

and

$$
\begin{aligned}
&\left|\partial\left(w_{1, \ldots, l, n}-w_{1, \ldots, l, m}\right) / \partial x\right|_{2, \tau} \text { and }\left\|w_{1, \ldots, l, n}-w_{1, \ldots, l, m}\right\|_{C(\mathbf{Q})} \\
& \leq \frac{2^{1 / 2}(l+1) !\left(k_{-}+k_{+}\right)}{k_{-}^{l+2}}\left(F_{0}+F_{1}\right) H_{1} \cdots H_{l} \\
& \cdot\left\{\left|k_{n}-k_{m}\right|_{0}+\frac{k_{-}}{l+1}\left(\sum_{i=1}^{l} \frac{\left|h_{i n}-h_{i m}\right|_{0}}{H_{i}}+\frac{\left\|f_{n}-f_{m}\right\|_{H^{2}(0, T)}}{F_{0}+F_{1}}\right)\right\}
\end{aligned}
$$

for any $\tau \in[0, T]$ and $m, n, l=1,2, \ldots$. 
From the last three estimates it follows that there exist functions $w_{1,2, \ldots, l}(x, t) \in$ $C(\mathbf{Q}), l=1,2,3, \ldots$, such that

$$
\begin{aligned}
& w_{1,2, \ldots, l, n}(x, t) \stackrel{C(\mathbf{Q})}{\longrightarrow} w_{1,2, \ldots, l}(x, t), \\
& \partial w_{1,2, \ldots, l, n}(x, t) / \partial x \stackrel{L^{2}(0,1)}{\longrightarrow} \partial w_{1,2, \ldots, l}(x, t) / \partial x \quad \text { for every } t \in[0, T] \text {, } \\
& \partial w_{1,2, \ldots, l, n}(x, t) / \partial t \stackrel{L^{2}(0,1)}{\longrightarrow} \partial w_{1,2, \ldots, l}(x, t) / \partial t \quad \text { for every } t \in[0, T]
\end{aligned}
$$

and

$$
\partial^{2} w_{1,2, \ldots, l, n}(x, t) / \partial t \partial x \stackrel{L^{2}(Q)}{\longrightarrow} \partial^{2} w_{1,2, \ldots, l}(x, t) / \partial t \partial x, \quad l=1,2,3, \ldots
$$

as $n \rightarrow \infty$.

The norms of $w_{1,2, \ldots, l}(x, t)$ and their derivatives also satisfy the same estimates as those for the norms $w_{1,2, \ldots, l, n}(x, t)$ and their derivatives (3.29). Moreover, it is clear that $w_{1,2, \ldots, l}(x, t)$ is the weak solution of $(3.12)$ corresponding to $\left\{k, h_{1}, \ldots, h_{l}, f\right\}$.

Now let the operators $B_{l}$ be defined as

$$
B_{l}(k) \cdot h_{1} h_{2} \cdots h_{l}=w_{1,2, \ldots, l}(x, t) \in C(\mathbf{Q})
$$

for any $k \in \Sigma, h_{i} \in C[0, T], i=1,2, \ldots, l$ and $l=1,2,3, \ldots$ Obviously, these operators are linear with respect to $h_{i}, i=1,2, \ldots, l$.

To prove the Fréchet differentiability of the operator $A_{1}$ up to an arbitrary order, one needs to verify the following equalities, one by one:

$$
\begin{aligned}
& \left|A_{1} \cdot k^{*}-A_{1} \cdot k-B_{1}(k) \cdot\left(k^{*}-k\right)\right|_{0}=O\left(\left|k^{*}-k\right|_{0}\right), \\
& \left|A_{1}^{\prime}\left(k^{*}\right) \cdot h_{1}-A_{1}^{\prime}(k) \cdot h_{1}-B_{2}(k) \cdot h_{1}\left(k^{*}-k\right)\right|_{0}=O\left(\left|k^{*}-k\right|_{0}\right), \\
& \left|A_{1}^{(l-1)}\left(k^{*}\right) \cdot h_{1} \cdots h_{l-1}-A_{1}^{(l-1)}(k) \cdot h_{1} \cdots h_{l-1}-B_{l}(k) \cdot h_{1} \cdots h_{l-1}\left(k^{*}-k\right)\right|_{0} \\
& =O\left(\left|k^{*}-k\right|_{0}\right) \text {, }
\end{aligned}
$$

In fact, upon using the results in Lemma 4 for their approximations, one obtains

$$
\left|u_{n}^{*}(x, t)-u_{n}(x, t)-w_{1 n}(x, t)\right| \leq 2^{1 / 2}\left(k_{-}+k_{+}\right) k_{-}^{-3}\|f\|_{H^{1}(0, T)}\left|k^{*}-k\right|_{0}^{2}
$$

and

$$
\begin{aligned}
& \left|w_{1,2, \ldots, l-1, n}^{*}(x, t)-w_{1,2, \ldots, l-1, n}(x, t)-w_{1,2, \ldots, l, n}(x, t)\right| \\
& \leq 2^{1 / 2}(l+1) !\left(k_{-}+k_{+}\right) k_{-}^{-l-2}\|f\|_{H^{1}(0, T)}\left|h_{1}\right|_{0} \cdots\left|h_{l-1}\right|_{0}\left|k^{*}-k\right|_{0}^{2}, \\
& \quad l=2,3, \ldots
\end{aligned}
$$

Hence, as $n \rightarrow \infty$, the above equalities hold. By the definition of the Fréchet differential, one has $A_{1}^{(l)}(k) \cdot h_{1} h_{2} \cdots h_{l}=B_{l}(k) \cdot h_{1} h_{2} \cdots h_{l}$ and

$$
A^{(l)}(k) \cdot h_{1} h_{2} \cdots h_{l}=w_{1,2, \ldots, l}(0, t)=-\int_{0}^{1} \partial\left(w_{1,2, \ldots, l}(x, t)\right) / \partial x d x \in C[0, T] .
$$

4. Convergence of the Iterative Solution of a GPST. Before proving the main convergence theorems, we need the following lemma from [12]. 
LEMMA 7. Let $\phi$ be an operator from a Banach space $X$ into another Banach space $Y$ and $\phi$ be Fréchet differentiable in $\Omega \subset X$. Let $N_{n}$ be a sequence of linear invertible operators from $X$ into $Y$ such that for given nonnegative constants $\lambda, \xi$, $\beta$ and $\eta$,

(i) $\left\|N_{n}^{-1}\right\| \leq \lambda$,

(ii) $\left\|N_{n}-\phi^{\prime}\left(k_{0}\right)\right\| \leq \xi$,

(iii) if $k^{*}, k$ are in the sphere $S\left(k_{0}, \rho\right) \subset \Omega$, then

$$
\left\|\phi\left(k^{*}\right)-\phi(k)-\phi^{\prime}\left(k_{0}\right) \cdot\left(k^{*}-k\right)\right\|_{Y} \leq \beta\left\|k^{*}-k\right\|_{X},
$$

(iv) $\left\|\phi\left(k_{0}\right)\right\|_{Y} \leq \eta$, and

(v) $\varsigma=\lambda(\beta+\xi)<1, r_{0}=\lambda \eta(1-\varsigma) \leq \rho$.

Then the iteration $k_{n+1}=k_{n}-N_{n}^{-1} \cdot \phi\left(k_{n}\right), n=0,1,2,3, \ldots$, is well defined and converges to a solution $\mathbf{k}$ of $\phi(k)=0$. Furthermore, $\left\|\mathbf{k}-k_{0}\right\|_{X} \leq r_{0}$, and $\mathbf{k}$ is the only solution contained in this sphere. The rate of convergence is given by $\left\|\mathbf{k}-k_{n}\right\|_{X} \leq r_{0} \varsigma^{n}$.

To put the special form of GPST described in Section 2 into the mathematical framework of Lemma 7, we let the operators $\phi(k)$ and $N_{n}$ be given by $(2.5)$ and (2.6), respectively, and let $X=C[0,1], Y=V_{0}=(C[0,1])^{*}$ and $\Omega=\Sigma$. By using the properties of the operator $A$ proved in the previous section it can be easily shown that $\phi(k)$ is Fréchet differentiable,

$$
\phi^{\prime}(k)=A^{\prime *}(k) \cdot A^{\prime}(k)+A^{\prime \prime *}(k) \cdot(A \cdot k-g)+\alpha^{2} B,
$$

and that there exist positive constants $L_{1}, L_{2}$ and $L_{3}$, depending only on $k_{-}, k_{+}$ and $\|f\|_{H^{1}(0, T)}$ such that

$$
\begin{gathered}
\left\|A^{\prime *}\left(k^{*}\right) \cdot\left(A \cdot k^{*}-g\right)-A^{\prime *}(k) \cdot(A \cdot k-g)\right\| \leq L_{1}\left|k^{*}-k\right|_{0}, \\
\left\|A^{\prime *}\left(k^{*}\right) \cdot A^{\prime}\left(k^{*}\right)-A^{\prime *}(k) \cdot A^{\prime}(k)\right\| \leq L_{2}\left|k^{*}-k\right|_{0}
\end{gathered}
$$

and

$$
\left\|A^{\prime \prime *}\left(k^{*}\right) \cdot\left(A \cdot k^{*}-g\right)-A^{\prime \prime *}(k) \cdot(A \cdot k-g)\right\| \leq L_{3}\left|k^{*}-k\right|_{0}
$$

for any $k^{*}, k \in \Sigma$. Hence Lemma 7 applied to the special form of GPST becomes the following lemma.

LEMMA 8. Suppose that there exist positive constants $\lambda$ and $\eta$ and $k_{0} \in \Sigma$ such that

$$
\begin{gathered}
\left\|\phi\left(k_{0}\right)\right\|_{Y} \leq \eta, \\
\left\|N_{0}^{-1}\right\|=\left\|\phi^{\prime}\left(k_{0}\right)^{-1}\right\| \leq \lambda
\end{gathered}
$$

and

$$
\lambda^{2} \eta \leq(4 C)^{-1}
$$

where $C=3 L_{2}+L_{3}$, with $L_{2}$ and $L_{3}$ being the Lipschitz constants of $A^{\prime *} \cdot A^{\prime}$ and $A^{\prime \prime *} \cdot(A k-g)$, respectively. Then the iterative sequence $\left\{k_{n}\right\}$ of $(2.6)$ converges to a solution $k_{\alpha}(x)$ of $\phi(k)=0$. Moreover, its rate of convergence is characterized by the estimate

$$
\left\|k_{\alpha}-k_{n}\right\|_{X}=r_{0} \varsigma^{n}
$$

where $r_{0}=\lambda \eta(1-C \lambda \rho)^{-1}$ and $\varsigma=\lambda\left(2 L_{2}+L_{3}\right) \rho\left(1-L_{2} \lambda \rho\right)^{-1}$. 
Proof. To show that the hypotheses (4.1)-(4.3) are equivalent to the hypotheses (i) $-(\mathrm{v})$ of Lemma 7 , we note first that (4.1) is equivalent to (iv). Next, let $\rho=$ $(2 \lambda C)^{-1}\left(1-\left(1-4 \lambda^{2} \eta C\right)^{1 / 2}\right)$; obviously, $\rho$ satisfies $\rho=\lambda \eta(1-\lambda \rho C)^{-1}$. From (4.3), $\lambda \rho C<\frac{1}{2}$. Let $S\left(k_{0}, \rho\right) \subset \Sigma$; then for any $k_{n} \in S\left(k_{0}, \rho\right)$ we have

$$
\begin{aligned}
\left\|N_{n}-\phi^{\prime}\left(k_{0}\right)\right\| & =\left\|N_{n}-N_{0}\right\|=\left\|A^{\prime *}\left(k_{n}\right) \cdot A^{\prime}\left(k_{n}\right)-A^{\prime *}\left(k_{0}\right) \cdot A^{\prime}\left(k_{0}\right)\right\| \\
& \leq L_{2}\left\|k_{n}-k_{0}\right\|_{X} \leq L_{2} \rho=\xi,
\end{aligned}
$$

which verifies (ii) and $\left\|N_{0}^{-1}\left(N_{n}-N_{0}\right)\right\| \leq L_{2} \lambda \rho \leq C \lambda \rho<\frac{1}{2}$ from (4.2). From [9], the operator $N_{n}$ is invertible and $\left\|N_{n}^{-1}\right\| \leq \lambda\left(1-L_{2} \lambda \rho\right)^{-1}=\lambda^{*}$, which implies (i).

For any $k \in S\left(k_{0}, \rho\right)$, one also has

$$
\begin{aligned}
\left\|\phi^{\prime}(k)-\phi^{\prime}\left(k_{0}\right)\right\| \leq & \left\|A^{\prime *}\left(k_{n}\right) \cdot A^{\prime}\left(k_{n}\right)-A^{\prime *}\left(k_{0}\right) \cdot A^{\prime}\left(k_{0}\right)\right\| \\
& +\left\|A^{\prime \prime *}\left(k_{n}\right) \cdot\left(A \cdot k_{n}-g\right)-A^{\prime * *}\left(k_{0}\right) \cdot\left(A \cdot k_{0}-g\right)\right\| \\
\leq & \left(L_{2}+L_{3}\right) \rho ;
\end{aligned}
$$

thus, for any $k^{*}, k \in S\left(k_{0}, \rho\right)$, one obtains

$$
\begin{aligned}
& \left\|\phi\left(k^{*}\right)-\phi(k)-\phi^{\prime}\left(k_{0}\right)\left(k^{*}-k\right)\right\|_{Y} \\
& \quad=\left\|\int_{0}^{1}\left\{\phi^{\prime}\left(k+t\left(k^{*}-k\right)\right)-\phi^{\prime}\left(k_{0}\right)\right\} d t \cdot\left(k^{*}-k\right)\right\|_{Y} \\
& \quad \leq\left(L_{2}+L_{3}\right)\left\|k^{*}-k\right\|_{X}=\beta\left\|k^{*}-k\right\|_{X},
\end{aligned}
$$

which is equivalent to (iii). Finally, let

$$
\varsigma=\lambda^{*}(\beta+\xi)=\lambda\left(2 L_{2}+L_{3}\right) \rho\left(1-L_{2} \lambda \rho\right)^{-1} .
$$

Then it is clear that $\zeta<1$ and $r_{0}=\lambda^{*} \eta(1-\zeta) \leq \lambda \eta(1-C \lambda \rho)^{-1}=\rho$, which implies (v). Hence, from Lemma 7, the conclusion of Lemma 8 is proved.

Now the main result is contained in the following theorem.

THEOREM. Assume that there exists a regularized solution $k_{\alpha}(x) \in \Sigma$ for $\alpha>0$ small enough such that $\phi\left(k_{\alpha}\right)=0$ and $\phi^{\prime}\left(k_{\alpha}\right)$ is invertible. Then there exists at least one sequence $\left\{k_{n}\right\}$ from (2.6) that converges to $k_{\alpha}(x)$. Moreover, the following error estimate holds, $\left\|k_{\alpha}-k_{n}\right\|_{X} \leq r_{0} \varsigma^{n}$, where $r_{0}$ and $\zeta<1$ are two positive constants depending only on $\alpha,\|B\|, L_{1}, L_{2}, L_{3}$ and $\left\|\phi^{\prime}\left(k_{\alpha}\right)^{-1}\right\|$.

Proof. To show that there exists a $k_{0} \in \Sigma$ such that the hypotheses (4.1)-(4.3) of Lemma 8 are satisfied, let $\left\|\phi^{\prime}\left(k_{\alpha}\right)^{-1}\right\|=\frac{1}{2} \lambda, \lambda>0$, and $\delta=\operatorname{Min}\left\{\lambda^{-1}\left(L_{2}+L_{3}\right)^{-1}\right.$, $\left.\frac{1}{4} \lambda^{-2} C^{-1}\left(L_{1}+\alpha^{2}\|B\|\right)^{-1}\right\}$. For any $k_{0} \in S\left(k_{\alpha}, \delta\right) \cap \Sigma$, one has

$$
\begin{aligned}
\left\|\phi\left(k_{0}\right)\right\|_{Y} & =\left\|\phi\left(k_{0}\right)-\phi\left(k_{\alpha}\right)\right\|_{Y} \\
& \leq\left\|A^{\prime *}\left(k_{0}\right) \cdot\left(A \cdot k_{0}-g\right)-A^{\prime *}\left(k_{\alpha}\right) \cdot(A \cdot k-g)\right\|+\alpha^{2}\left\|B \cdot k_{0}-B \cdot k_{\alpha}\right\| \\
& \leq\left(L_{1}+\alpha^{2}\|B\|\right)\left\|k_{0}-k_{\alpha}\right\|_{X} \leq\left(L_{1}+\alpha^{2}\|B\|\right) \delta=\eta,
\end{aligned}
$$

i.e., (4.1) is satisfied. Since

$$
\begin{aligned}
\left\|\phi^{\prime}\left(k_{0}\right)-\phi^{\prime}\left(k_{\alpha}\right)\right\| \leq & \left\|A^{\prime *}\left(k_{0}\right) \cdot A^{\prime}\left(k_{0}\right)-A^{\prime *}\left(k_{\alpha}\right) \cdot A^{\prime}\left(k_{\alpha}\right)\right\| \\
& +\left\|A^{\prime *}\left(k_{0}\right) \cdot\left(A \cdot k_{0}-g\right)-A^{\prime * *}\left(k_{\alpha}\right) \cdot\left(A \cdot k_{\alpha}-g\right)\right\| \\
\leq & \left(L_{2}+L_{3}\right)\left\|k_{0}-k_{\alpha}\right\|_{X} \leq\left(L_{2}+L_{3}\right) \delta \leq \lambda^{-1}
\end{aligned}
$$


and $\left\|\phi^{\prime}\left(k_{\alpha}\right)^{-1}\right\|=\frac{1}{2} \lambda$, one can show that $\phi\left(k_{0}\right)$ is invertible and $\left\|\phi^{\prime}\left(k_{0}\right)^{-1}\right\| \leq$ $\lambda$, i.e., (4.2) is satisfied. Next, from (4.1) and (4.2) it follows that $\lambda^{2} \eta=$ $\lambda^{2}\left(L_{1}+\alpha^{2}\|B\|\right) \delta \leq \frac{1}{4} C^{-1}$, i.e., (4.3) is satisfied. Finally, Lemma 8 yields the results of the theorem.

Acknowledgment. One of the authors, X. Y. Liu, was partially supported by the Committee on Educational Exchange with China at the State University of New York at Stony Brook as the 1983-1984 Fong Shu-chuen Fellow and by Chevron Oil Field Research Company at La Habra, California.

Department of Mathematics

Peking University

Beijing, People's Republic of China

Department of Applied Mathematics and Statistics

State University of New York at Stony Brook

Stony Brook, New York 11794

1. Y. M. CHEN, "Generalized pulse-spectrum technique (GPST) for solving problems in parameter identification," Proc. U.S.-China Workshop on Advances in Computational Engineering Mechs., Dalian, China, Sept. 5-9, 1983.

2. Y. M. CHEN \& D. S. TSIEN, "A numerical algorithm for remote sensing of density profiles of a simple ocean model by acoustic pulses," J. Comput. Phys., v. 25, 1977, pp. 366-385.

3. Y. M. CHEN \& J. Q. LIU, "A numerical algorithm for remote sensing of thermal conductivity," J. Comput. Phys., v. 43, 1981, pp. 315-326.

4. Y. M. CHEN \& J. Q. LIU, "A numerical algorithm for solving inverse problems of twodimensional wave equation," J. Comput. Phys., v. 50, 1983, pp. 193-208.

5. Y. M. CHEN \& J. Q. LIU, "An iterative numerical algorithm for solving multi-parameter inverse problems of evolutional partial differential equations," J. Comput. Phys., v. 53, 1984, pp. 429-442.

6. Y. M. ChEN \& G. Q. XIE, "A numerical method for simultaneous determination of bulk modulus, shear modulus and density variations for nondestructive evaluation," Nondestructive Testing Communication, v. 1, 1984, pp. 125-135.

7. Y. M. CHEN \& G. Q. XIE, "An iterative method for simultaneous determination of bulk and shear moduli and density variation," J. Comput. Phys., v. 62, 1986, pp. 143-163.

8. A. Friedman, Partial Differential Equations of Parabolic Type, Prentice-Hall, Englewood Cliffs, N. J., 1964.

9. L. A. Kantorovich \& G. P. AKIlov, Functional Analysis in Normed Spaces, Pergamon Press, Oxford, 1964.

10. J. Q. LIU \& Y. M. CHEN, "An iterative algorithm for solving inverse problems of twodimensional diffusion equations," SIAM J. Sci. Statist. Comput., v. 5, 1984, pp. 255-269.

11. X. Y. LIU \& Y. M. CHEN, "A generalized pulse-spectrum technique for determining timedependent coefficients of one-dimensional diffusion equations," SIAM J. Sci. Statist. Comput., v. 8, 1987, pp. 436-445.

12. V. PEREYRA, "Iterative methods for solving nonlinear least squares problems," SIAM J. Numer. Anal., v. 4, 1967, pp. 27-36.

13. Y. N. TANG \& Y. M. CHEN, "Application of GPST algorithm to history matching of singlephase simulator models," Advances in Computer Methods for Partial Differential Equations V (R. Vichnevetsky and R. Stepleman, eds.), IMACS, 1984, pp. 433-439.

14. A. N. Tikhonov \& V. Y. ARsenin, Solutions of Ill-Posed Problems, Wiley, New York, 1977.

15. G. Q. XIE \& Y. M. CHEN, "A modified pulse-spectrum technique for solving inverse problems of two-dimensional elastic wave equation," Appl. Numer. Math., v. 1, 1985, pp. 217-237.

16. G. Q. XIE, Y. M. CHEN \& X. Y. LIU, "Convergence of a generalized pulse-spectrum technique (GPST) for inverse problems of 1-D evolutional partial differential equations," SIAM J. Numer. Anal. (To appear.) 